Path Independence, Rationality, and Social Choice

Author(s): Charles R. Plott

Source: Econometrica, Vol. 41, No. 6 (Nov., 1973), pp. 1075-1091

Published by: The Econometric Society

Stable URL: http://www.jstor.org/stable/1914037

Accessed: 24/09/2010 18:08

Your use of the JSTOR archive indicates your acceptance of JSTOR's Terms and Conditions of Use, available at http://www.jstor.org/page/info/about/policies/terms.jsp. JSTOR's Terms and Conditions of Use provides, in part, that unless you have obtained prior permission, you may not download an entire issue of a journal or multiple copies of articles, and you may use content in the JSTOR archive only for your personal, non-commercial use.

Please contact the publisher regarding any further use of this work. Publisher contact information may be obtained at http://www.jstor.org/action/showPublisher?publisherCode=econosoc.

Each copy of any part of a JSTOR transmission must contain the same copyright notice that appears on the screen or printed page of such transmission.

JSTOR is a not-for-profit service that helps scholars, researchers, and students discover, use, and build upon a wide range of content in a trusted digital archive. We use information technology and tools to increase productivity and facilitate new forms of scholarship. For more information about JSTOR, please contact support@jstor.org. 


\title{
PATH INDEPENDENCE, RATIONALITY, AND SOCIAL CHOICE
}

\author{
By Charles R. PlotT ${ }^{1}$
}

\begin{abstract}
The paper provides several axiomatizations of the concept of "path independence" as applied to choice functions defined over finite sets. The axioms are discussed in terms of their relationship to "rationality" postulates and their meaning with respect to social choice models.
\end{abstract}

\section{INTRODUCTION}

IN ANSWER to critics of the first edition of Social Choice and Individual Values, Arrow advanced in the second edition a justification for imposing his "consistency" or "rationality" conditions which had not previously appeared, explicitly, in the social choice literature $[2$, p. 120]. He argued that the rationality conditions were necessary in order for social choices to be independent of the path of choice. He provided no real elaboration on the point. Perhaps he felt no explanation was necessary, since much of the social choice literature, especially those papers which deal with cycles, implicitly place a premium on some type of path-independence property.

The purpose of this paper is to report some results which bear on the meaning and usefulness of this type of property. Specifically, it is shown that path independence is implied by, but does not imply rational choice. The importance of the observation is threefold. First, if path independence, rather than rationality, is desired as a property of social choice, the stronger rationality conditions need not be imposed. One result of this relaxation is that the immediate impossibility result discovered by Arrow is avoided. Welfare economists then are free to explore the possible applications of the tools he provided. Secondly, the observations made raise issues pertaining to the reasons for investigating mathematical properties like path independence in the first place. Thirdly, it is shown that the lines which separate rationality properties, which induce immediate impossibility results, from path-independence properties are very thinly drawn.

We will proceed as follows. Immediately below, in Section 2, a survey of the interpretations of the symbols is given. A glossary is also added at the end. Section 3 provides a brief summary of the arguments which have been advanced in support of "rationality conditions" in the case of social choice. These are presented in order that they can be separated from those arguments which are

\footnotetext{
${ }^{1}$ The author gratefully acknowledges the financial support supplied by National Science Foundation Grant GS36214 and Resources for the Future. In addition, he wishes to thank Robert P. Parks who found an error in the proof of Theorem 3 in an early draft, and also A. K. Sen and Joel Franklin who provided useful comments. Of course, responsibility for error remains with the author.
} 
advanced in favor of the path-independence properties. Section 4 contains a discussion of what I consider to be the major "path-independence" type axiom offered here. Its motivation, interpretation, simple mathematical structure, and relationship with rationality conditions are outlined. Section 5 contains two additional axioms. These two axioms are presented as attempts to capture the essence of a second motivation for path independence, which, I suspect, is closer to that which Arrow had in mind. These two axioms are discussed and connected to rationality conditions. The final section is a discussion of the general possibility theorem in view of the results presented here.

\section{NOTATION AND SYMBOLS}

Let the set of all conceivable "social states" be designated as $E$. Each element of $E$ is taken as a complete description of the relevant universe. What is "relevant" depends upon the model. In terms of some broad sociopolitical-economic model, an element of $E$ could be a complete description of the consumption and production activities of each economic agent (including receiving and discharging pollutants), and/or bills passed by the legislature, and/or the resolution of every conflict situation.

Consider an arbitrary subset $v$ of $E$. We say the "social choice" over $v$ is a subset of $v$. That is, $C(v) \subset v$ where $C(v)$ is the set of "chosen" elements. In case $v$ is a consumption possibility set for an economy, then $C(v)$ could be the competitive equilibria, or the core of the economy, or possibly even a von NeumannMorgenstern solution. The point is, $C(v)$ can be viewed as the set of outcomes (allowing for the possibility of non-unique solutions) ${ }^{2}$ of some decision process, whether the process is economic, sociological, or political. The only requirement is that the function $C(v)$ be dictated by the socio-economic laws governing the process. ${ }^{3}$ For purposes of exposition, the set $v$ will be called the "agenda."

A set of $v$, or a family of subsets of $E, V=\left\{v_{1}, \ldots, v_{m}\right\}$ will be called the "admissible agenda." This is the collection of sets for which the social choice operates. For example, for $E=\{x, y, z\}$ it could be the case that $V=\{\{x, y\},\{x, z\},\{x, y, z\}\}$. In a limited sense, it is the set of "situations" which might face a society. In the case of an economy, it would be the family of consumption possibility sets, any one of which might conceivably occur. In the case of a legislature, it is a family of sets of proposals which a legislature might face. The precise nature of the admissible agenda is dependent upon the problem. We assume $C(v) \neq \varnothing$ for any admissible $v$, except, of course, for $v=\varnothing$, as part of the structure of the problem.

There may be parameters on the social choice function other than $v$. For example, the choice may depend not only on $v$ but also on individual attitudes about the elements of $E$. In case individuals have "preferences" over $E$, we could

\footnotetext{
${ }^{2}$ For a discussion of game-theoretic interpretations of such choice structures, see $[9,17$, and 18].

${ }^{3} \mathrm{We}$ can view the choice function as either itself a model of a process or a model of a model of a process. In either case, the "facts" directly or indirectly are taken as giving the choice function its form. For a review of many of the models that have been developed, see Plott [9].
} 
designate $R_{i}$ as the preference relation of the $i$ th individual and, where there are $n$ individuals, write the choice function as $C\left(v, R_{1}, \ldots, R_{n}\right) \subset v$. This would indicate that the choice from $v$ is a subset of $v$, but the particular subset chosen depends upon the vector of individual preference relations. Where $D$ is a set of "admissible" vectors of individual preference relations, the 4-tuple $\langle D, E, V, C(\cdot)\rangle$ has been called a constitution. ${ }^{4}$

The analysis here will proceed, for the most part, on the assumption that individual tastes are fixed. Consequently, the additional notation will be dropped. That is, we shall assume individual tastes and other parameters to be fixed even though the agenda may change. The notation $C(v)$ is simpler than $C\left(v, \bar{R}_{1}, \bar{R}_{2}, \ldots, \bar{R}_{n}\right)$ where the bar indicates a constant. Since individual preferences will be assumed fixed, the broad construction of a constitution is unnecessary. The relevant information will be carried simply as $\langle V, C(\cdot)\rangle$, where $V$ is the family of admissible agenda and $C(\cdot)$ is the choice function.

Of the many aspects of the pair $\langle V, C(\cdot)\rangle$ that could be investigated, only one is of interest here - the rationality properties of $\langle V, C(\cdot)\rangle$. Technically speaking, the choice $C(\cdot)$ is rational in case there exists a "preference" relation that "rationalizes" it : that is, in case there exists a binary relation, $R$, such that for ever $y$ admissible $v$, the choice $C(v)$ is the set of "maximal" elements in $v$, according to the binary relation $R .{ }^{5}$ Consider the example $V=\{\{x, y\},\{y, z\}\}$ and suppose $C(\{x, y\})=\{x\}$ and $C(\{y, z\})=\{y, z\}$. Now $x P y, x P z, y I z$ is a transitive rationalization while $x P y, y I z, x I z$ is an intransitive rationalization of the same data. ${ }^{6}$

Notice the concept of rational choice does not presuppose that the rationalization of any given data is necessarily transitive. In fact, the concept presupposes no properties of the rationalization at all, other than that it must be a binary relation. Of course, the given data themselves will induce properties on rationalizations. Classifications of rationalizations, or "degrees of rationality," such as total, reflexive, transitive, etc., can be listed. ${ }^{7}$ Analysis could then proceed on the relationships between data that would guarantee that any rationalization would be in one or more of these various classes. This will be the procedure used here, but interest will be limited to simply one or two types of rationalizations.

\section{WHY RATIONAL SOCIAL CHOICE?}

In a very real sense the "degree" of rationality imposed on or required in a constitution depends upon one's view of society and, more importantly, one's view of the purposes of social choice theory. Unfortunately, the question "why rational

\footnotetext{
${ }^{4}$ For the development and discussion of this construction, see [9].

${ }^{5}$ Formally, a binary relation $R$ rationalizes, or is a rationalization of, $\langle V, C(\cdot)\rangle$ in case $(\forall v)_{v \in V} C(v)=$ $\left\{x \in v:(\forall y)_{y \in v} x R y\right\}$. If $R$ is a rationalization of $\langle V, C(\cdot)\rangle$, it is said to induce $C(\cdot)$ over $V$.

${ }^{6}$ The relations of $\alpha P \beta$ and $\alpha I \beta$ are defined as $\alpha R \beta \& \sim \beta R \alpha$, and $\alpha R \beta \& \beta R \alpha$, respectively, where $R$ is the rationalization. In this particular example we have from the observed choices that any rationalization must have the properties $x R y$ and $\sim y R x$ and $y R z$ and $z R y$. For this example, any binary relation with these properties is a rationalization. The second rationalization given in the text is intransitive, since $y R z \& z R x \neq y R x$.

${ }^{7}$ See Plott [9] and Richter [13].
} 
social choice?" is seldom discussed. Then, when the issue is raised, there is a remarkable tendency for writers to "talk past" each other. It is as though each writer finds the answer so "obvious" that he feels little need to be clear on the topic when addressing his colleagues or reading what his colleagues have to say on the subject. Consequently, many potentially serious disagreements never surface.

The case of individual choice is rather easy to ur.derstand. Individual choices, for some reason or another, are often consistent with some preference relation over the commodity set, and Richter's $W$-axiom [13] would appear to be on the order of a law. The case of social choice appears to be different. There seems to be nothing natural at all about rational social choice. In fact, it was in the answer to Buchanan's [4] charge that Arrow was illegitimately transferring properties of individual choice to social choice, that the issue of path independence surfaced in the first place.

Evidently, some view society as an organism (thinking? feeling?) with a "personality," "preference," or "interest" all of its own which is independent of the preferences of individuals. Of course, if one has such a view, requirements of "rationality" would be a natural thing to incorporate in a social choice model. The "degree" of rationality exhibited by choice would, presumably, be the same as that of other "independent" agents, such as individuals, unless, of course, one sees that fellow named Society as having a "deviant personality."

Many of the efforts in the literature are attempts to define or abstractly quantify something called a "social preference." Operationally, it is difficult to distinguish efforts which are motivated from this point of view from those having the motivation outlined in the paragraph above. ${ }^{8}$ The reason for the difficulty in making the distinction at the operational level is that no one has outlined a convention for determining the bases on which the "social preference" can be judged to be successfully defined. Arrow, on the one hand, appears to be motivated at times by the simple personal feeling that acceptable social choice processes must necessarily be characterized by some sort of consistency. Similarly, Sen [15] views an identification of the "most preferred" to be some sort of inherent precondition to choice. The ground rules for the discussion have not been set.

If one is to speak of social choices as the "most preferred," the "best," or the "optimal," then just from the meaning of the words alone ${ }^{9}$ it would follow that choice must be consistent with some binary relation. Of course, there are many candidates for properties required of the binary relation (total? quasi-transitive? transitive?), and this procedure apparently provides no basis for choosing from among them. It is also true that this view never really supplies a reason for justifying

\footnotetext{
${ }^{8}$ I suppose those who are of the persuasion outlined in the first paragraph could proceed by introspection-" "what would I do if I were it?" - "it" being society.

"Suppose $x$ is judged "fair" when the possibilities are $(x, y), y$ is judged "fair" when the possibilities are $(z, y)$, and $z$ is judged "fair" when the possibilities are $(x, z)$. This result does not seem so "odd" to some, whereas to substitute "better" for "fair" would be in violation of their understanding of the words "better" and "best." Put another way, to some, what is "fair" may depend upon the agenda. This is analogous to an argument for the possibility of employing a different social welfare function for every production possibility set.
} 
the demand that social choices be restricted to those which are consistent with some concept of "best" in the first place. I suspect that those who appear to subscribe to this point of view feel that the ethic, "the alternatives most preferred by society should be chosen," would be universally accepted if the "proper" definition of "social preference" were provided.

A procedure of demanding the existence of an indicator of "social welfare" which is independent of the particular production possibilities set, has been a basic tenet of almost all welfare economics stemming from Bergson. ${ }^{10}$ Indeed, the directions as given by the welfare function are frequently taken to be the point from which the applied welfare economist departs. Exactly why the directions must necessarily come in this particular form is not thoroughly discussed. I suspect there has been a presupposition that the definition of welfare would in some sense represent an ethic and that ethics must necessarily be of that mathematical form.

The paper here is not the place to explore the merits or demerits of all these views. Only those which might serve as a basis for formalizing some concept of "path independence" will be examined. The views above are outlined and isolated so that they need not be interjected here as the argument proceeds. It should be pointed out now that part of the discussion which follows presupposes a conceptualization as to the purpose of social choice theory which differs slightly from that presupposed in the discussions above. The problem is not viewed here as one of defining a social preference. Rather, it is seen as one of designing processes for which the choice function, $C(v)$, has "nice" features. The focus is, in part, upon whether or not the institutions available offer sufficient flexibility to permit a "successful" design.

In the next two sections we will present two separate classes of arguments. Both are motivated by the idea that social choice should, in some sense, be independent of previous choices. Throughout the paper we will assume that $E$ is finite and $C(\cdot) \neq \varnothing$ for all nonempty subsets of $E$.

\section{DIVIDE AND CONQUER}

The first motivation stems from the observation that the process of choosing, from a dynamic point of view, frequently proceeds in a type of "divide and conquer" manner. The alternatives are "split up" into smaller sets, a choice is made over each of these sets, the chosen elements are collected, and then a choice is made from them. Path independence, in this case, would mean that the final

${ }^{10}$ See [9, p. 116] for a formal characterization of the Bergson-Samuelson social welfare function. The contention there is that the Bergson-Samuelson welfare function is a constitution satisfying all of Arrow's conditions, but defined only for a single vector of individual preferences. For every vector of individual preferences one must define a new constitution or social welfare function with the requisite qualities. Each would satisfy all of Arrow's conditions except the first. This is a rather awkward distinction to make, except in the interest of the history of ideas, since, in a world of changing tastes a family of constitutions must be characterized. All of the results which follow from the Bergson welfare function, as well as the spirit of the idea, are retained by simply discussing a single constitution which is defined for all vectors of individual preferences but does not satisfy Arrow's independence of irrelevant alternatives. 
result would be independent of the way the alternatives were initially divided up for consideration.

Clearly, this could be a description of either individual choice or social choice. The case of social choice might be of special interest, however, where the outcomes that should result when the process is functioning "correctly" have been prespecified and the welfare economist is charged with the responsibility of creating or repairing the process in a manner which assures that the pre-specified outcomes will, in fact, occur. A problem of path independence can arise in this case from what appears to be fundamental limitations on institutions. For example, the concept of "majority rule," inherently, refers to a choice over a two-element set. The idea of a proposal either "passing" or "failing" is, at base, binary. Without pursuing this much further, one can easily imagine a group restricting deliberations to four or five issues at a time because of overriding considerations relating to communication costs and other logistical problems. If the institutions a welfare economist has at his disposal for the creation of social choice processes can only operate on "small" sets, how then can he create a choosing process which will handle a "non-small" set? The answer is simple. If the institutions will operate on any "small" set, he can apply them repeatedly. He would thereby "extend" choice from smaller sets to a choice over the non-small set. For example, if his institutions operated on any set with five elements or less, he would split a larger set into smaller sets of five elements or less and then proceed as above to choose, collect choices, and choose again.

Now, if he can design the process so that the outcomes are independent of the initial groupings, that parameter, "path," is one less that he has to worry about. Suppose individuals 1,2 , and 3 have, respectively, preference relations $x>y>z$, $y \succ z \succ x$, and $z \succ x>y$. Suppose further that the process he is to create, in this case, must yield $x$ as the outcome. If he can only use the binary process of majority rule and if he has no control over the parameter, "path," then it would appear that he has an unsolvable problem.

Let us proceed now, and make the idea of path independence formal. We proceed as follows. Assume $E$ is a finite set. Let $V=\left\{v_{1}, \ldots, v_{m}\right\}$ be a family of subsets of $E$ and for all $S, S \subset E$, define $V_{S}=\left\{V: \bigcup_{v \in V} v=S\right\}$. Assume $v \subset E$ $\& v \neq \varnothing \Rightarrow C(v) \neq \varnothing$.

INDEPENDENCE OF PATH (I.P.):

$$
\forall S_{S \subset E}\left\{V \in V_{S} \& V^{\prime} \in V_{S} \Rightarrow C\left(\bigcup_{v \in V} C(v)\right)=C\left(\bigcup_{v^{\prime} \in V^{\prime}} C\left(v^{\prime}\right)\right)\right\} .
$$

The axiom says we can choose over $S$ directly or arbitrarily segment $S$, choose over the parts, and then choose over the choices without changing the ultimate result. Notice that the property holds for all $S$ so one can further refine the segments without worry.

Before proceeding further, it would perhaps be instructive to make some remarks which will help the reader understand the structure of the axiom. These first two remarks establish that I.P. alone is completely divorced from the concept of social rationality. 
REMARK 1: Not all $C(\cdot)$ which satisfy I.P. are rational.

Proof: We need only consider the example for $E=\{x, y, z\}, C(\{x, y\})=\{x, y\}$, $C(\{y, z\})=\{y, z\}, C(\{x, z\})=\{x, z\}$, and $C(\{x, y, z\})=\{x, y\}$. From the twoelement choices, we see that the only possible rationalization (where $\gamma I \beta \Leftrightarrow \gamma R \beta$ $\& \beta R \gamma)$ is $x \operatorname{III} I z x^{11}{ }^{11}$ However, for this binary relation, the maximal elements of $\{x, y, z\}$ are $\{x, y, z\}$, which is inconsistent with $C(\{x, y, z\})=\{x, y\}$. Consequently, no rationalization exists.

We must now show that the example satisfies I.P. We can give an intuitive argument that I.P. is satisfied by simply noting $C(\{C(\{x, y\}), z\})=C(\{C(\{x, z\}), y\})=$ $C(\{C(\{y, z\}), x\})=C(\{x, y, z\})=\{x, y\}$. In order to actually establish that I.P. is satisfied, we must allow for "repeated" and "overlapping" $v$ in $V$.

Suppose $S \neq E$. We have, by the construction of $C(\cdot)$ in this particular example and definition of $V_{S}$,

$$
(\forall V)_{V \in V_{S}}\left[C\left(\bigcup_{v \in V} C(v)\right)=C\left(\bigcup_{v \in V} v\right)=C(S)=S\right] .
$$

Suppose $S=E$. By construction $[x \in S \Rightarrow x \in C(S)] \&[y \in S \Rightarrow y \in C(S)]$, and by definition, $V \in V_{E} \Rightarrow\left[\bigcup_{v \in V} v=E\right]$; consequently we know, for $V \in V_{E},\{x, y\} \subset$ $\bigcup_{v \in V} C(v)$. If $z \in \bigcup_{v \in V} C(v)$, we have $C\left(\bigcup_{v \in V} C(v)\right)=C(\{x, y, z\})=\{x, y\}$. If $z \notin \bigcup_{v \in V} C(v)$, we have $C\left(\bigcup_{v \in V} C(v)\right)=C(\{x, y\})=\{x, y\}$. We conclude

$$
(\forall V)_{V \in V_{E}}\left[C\left(\bigcup_{v \in V} C(v)\right)=\{x, y\}\right] .
$$

Statements (1) and (2) together establish that the example satisfies I.P.

Q.E.D.

ReMarK 2: A choice function, $C(\cdot)$, can satisfy I.P. and have a total, reflexive rationalization without the relation being transitive.

Proof: Again we need only consider an example for $E=\{x, y, z\}$. Let $C(\{x, y\})=\{x, y\}, C(\{y, z\})=\{y, z\}, C(\{x, z\})=\{z\}$, and $C(\{x, y, z\})=\{y, z\}$. The unique total, reflexive rationalization is (when $\alpha P \beta \Leftrightarrow \alpha R \beta \& \sim \beta R \alpha$ ) $x I y I z, z P x$. Since $x R y \& y R z \not x R z$, this rationalization is not transitive.

We now show the example satisfies I.P. Again, an intuitive reason can be obtained by recalling that for any single element $\{\alpha\}, C(\{\alpha\})=\{\alpha\}$, and by noticing that

$$
\begin{aligned}
C(\{C(\{x, y\}), z\}) & =C(\{C(\{y, z\}, x\})=C(\{C(\{x, z\}), y\}) \\
& =C(\{x, y, z\})=\{y, z\} .
\end{aligned}
$$

In order to establish the result, we first note

$$
(\forall S)_{\substack{S \neq E \\ S \neq\{x, z\}}} C(S)=S
$$

${ }^{11}$ Read this $x I y$ and $y I z$ and $z I x$, etc. 
so we can state that,

$$
(\forall S)_{\substack{S \neq E \\ S \neq\{x, z\}}}(\forall V)_{V \in V S}\left[C\left(\bigcup_{v \in V} C(v)\right)=S\right]
$$

Furthermore,

$$
(\forall V)_{V \in V\{x, z\}}\left[C\left(\bigcup_{v \in V} C(v)\right)=\{z\}\right]
$$

For $S=E$ we have, for $V \in V_{E},\{y, z\} \subset \bigcup_{v \in V} C(v)$. If $x \in \bigcup_{v \in V} C(v)$, we have $C\left(\bigcup_{v \in V} C(v)\right)=C(\{x, y, z\})=\{y, z\}$. If $x \notin \bigcup_{v \in V} C(v)$, we have $C\left(\bigcup_{v \in V} C(v)\right)=$ $C(\{z, y\})=\{y, z\}$. So

$$
(\forall V)_{V \in V_{E}}\left[C\left(\bigcup_{v \in V} C(v)\right)=\{y, z\}\right] .
$$

Chaining together (3), (4), and (5) we have a statement of I.P. The example thus satisfies I.P. and the result is established.

Q.E.D.

The simple structure of the axiom is exposed by Theorem 1, below. It indicates that the three statements I.P., I.P.', and I.P." are all equivalent. The statement I.P.' is of interest because it is really the simple way to state the axiom. It says that one only need look, for mathematical purposes, to two-set families. The statement I.P." is of interest because of its own independent interpretation. That axiom says you can proceed by comparing with "winners" alternatives which have never been in a previous contest.

THEOREM 1: The following two axioms are each equivalent to I.P.:

$$
\left(\forall v_{1}\right)_{v_{1} \subset E}\left(\forall v_{2}\right)_{v_{2} \subset E} C\left(C\left(v_{1}\right) \cup C\left(v_{2}\right)\right)=C\left(v_{1} \cup v_{2}\right)
$$

and

(I.P.") $\quad\left(\forall v_{1}\right)_{v_{1} \subset E}\left(\forall v_{2}\right)_{v_{2} \subset E} C\left(v_{s} \cup C\left(v_{2}\right)\right)=C\left(v_{1} \cup v_{2}\right)$.

In order to prove this theorem we need two lemmas.

Lemma 1: If $C(\cdot)$ satisfies I.P.' and if $v \subset C\left(v^{*}\right)$ for some $v^{*}$, then $C(v)=v$.

Proof: Suppose $v \subset C\left(v^{*}\right)$ but $\hat{x} \in v$ and $\hat{x} \notin C(v)$. Now, since $v \subset v^{*}$, we have $\left(v^{*} \backslash v\right) \cup v=v^{*}$ and so we can conclude that $\hat{x} \in C\left(v^{*}\right)=C\left(C\left(v^{*} \backslash v\right) \cup C(v)\right)$ by application of I.P.' But $\hat{x} \notin C\left(v^{*} \backslash v\right)$ and $\hat{x} \notin C(v)$ by assumption, so $\hat{x} \notin C\left(C\left(v^{*} \backslash v\right)\right.$ $\cup C(v)$ ) which is a contradiction. (Note only disjoint $v_{1}$ and $v_{2}$ were used, so a little stronger theorem was proved than was stated.) 
Lemma 2: Suppose $C(\cdot)$ satisfies I.P.'; then

$$
(\forall S)_{S \subset E} C(S) \subset C\left(\bigcup_{v \in V} C(v)\right)
$$

for any $V$ such that $\bigcup_{v \in V} v=S$.

Proof: Suppose $C(\cdot)$ satisfies I.P.', $\hat{x} \in C(S)$, and $\hat{x} \notin C\left(\bigcup_{v \in V} C(v)\right)$ for some $S$ and some $V$ such that $\bigcup_{v \in V} v=S$. We see that, in this case, $\hat{x} \notin \bigcup_{v \in V} C(v)$. Otherwise, since $\left[S \backslash \bigcup_{v \in V} C(v)\right] \cup\left[\bigcup_{v \in V} C(v)\right]=S$, and since I.P.' is satisfied, we have $\hat{x} \in C(S)=C\left(C\left(S \backslash \bigcup_{v \in V} C(v)\right) \cup C\left(\bigcup_{v \in V} C(v)\right)\right.$ which implies, contrary to assumption, that $\hat{x} \in C\left(\bigcup_{v \in V} C(v)\right)$. We see also that $x \notin\left[S \backslash \bigcup_{v \in V} C(v)\right]$. If this were not so we could pick any $\hat{v} \in V$ with $\hat{x} \in \hat{v}$, apply I.P.' to get $\hat{x} \in C(S)=C(C(S \backslash \hat{v}) \cup C(\hat{v}))$ and note the contradiction with $\hat{x} \notin C(\hat{v})$ which is true from the conclusion above that $x \notin \bigcup_{v \in V} C(v)$.

Summarizing, we see $\hat{x} \notin\left\{\left[\bigcup_{v \in V} C(v)\right] \cup\left[S \backslash \bigcup_{v \in V} C(v)\right]\right\}$. But this contradicts the fact that $\hat{x} \in S=\left\{\left[\bigcup_{v \in V} C(v)\right] \cup\left[S \backslash \bigcup_{v \in V} C(v)\right]\right\}$. We conclude that the assumption $\hat{x} \notin C\left(\bigcup_{v \in V} C(v)\right)$ is false and the lemma is proved Q.E.D.

Proof of Theorem 1 : Consider first I.P.' If $C(\cdot)$ satisfies I.P., it clearly satisfies I.P.' Suppose $C(\cdot)$ satisfies I.P.' Consider any $S$ and any $V$ such that $\bigcup_{v \in V} v=S$. Clearly $S=\bigcup_{v \in V} v=\left\{\left[\bigcup_{v \in V} v\right] \cup\left[\bigcup_{v \in V} C(v)\right]\right\}$ so application of I.P.' yields $C(S)=C\left(C\left(\bigcup_{v \in V} v\right) \cup C\left(\bigcup_{v \in V} C(v)\right)\right)$, or simply, $C(S)=C\left(C(S) \cup C\left(\bigcup_{v \in V} C(v)\right)\right)$. Now apply Lemma 2 to get $C(S)=C\left(C\left(\bigcup_{v \in V} C(v)\right)\right)$ and Lemma 1 to get $C(S)=$ $C\left(\bigcup_{v \in V} C(v)\right)$ which is, since $S$ and $V$ were arbitrary, a statement of I.P. Therefore, $C(\cdot)$ satisfies I.P.

Consider now I.P." Let $C(\cdot)$ satisfy I.P. We have then, for all $v_{1}$ and $v_{2}, C\left(v_{1} \cup\right.$ $\left.v_{2}\right)=C\left(C\left(v_{1}\right) \cup C\left(v_{2}\right)\right)$. Apply Lemma 1 to get $C\left(v_{1} \cup v_{2}\right)=C\left(C\left(v_{1}\right) \cup C\left(C\left(v_{2}\right)\right)\right)$ and from the definition of I.P. we get

$$
\left(\forall v_{1}\right)_{v_{1} \subset E}\left(\forall v_{2}\right)_{v_{2} \subset E}\left[C\left(v_{1} \cup v_{2}\right)=C\left(v_{1} \cup C\left(v_{2}\right)\right)\right],
$$

which is a statement of I.P."

Suppose now that $C(\cdot)$ satisfies I.P." We have then $\left(\forall v_{1}\right)\left(\forall v_{2}\right)\left[C\left(v_{1} \cup v_{2}\right)=\right.$ $\left.C\left(v_{1} \cup C\left(v_{2}\right)\right)\right]$. Let $\hat{v}_{1}=v_{1}$ and $\hat{v}_{2}=C\left(v_{2}\right)$, and apply I.P.' to get $C\left(v_{1} \cup v_{2}\right)=$ $C\left(\hat{v}_{1} \cup \hat{v}_{2}\right)=C\left(C\left(\hat{v}_{1}\right) \cup \hat{v}_{2}\right)=C\left(C\left(v_{1}\right) \cup C\left(v_{2}\right)\right)$ which is, since $v_{1}$ and $v_{2}$ were unrestricted, a statement of I.P.' We have already shown that I.P.' implies I.P., so we are finished.

Q.E.D.

The mathematical structure underlying path independence concepts such as these, appears to be, at this time, the associative law. The following theorem makes an appropriate connection with something about which a lot is known from a mathematical point of view. The connection made here might provide the proper avenue to follow if one wants to drop the finiteness restriction or perform some other generalization. 
THEOREM 2: Define $v_{1} \cdot v_{2} \equiv C\left(C\left(v_{1}\right) \cup C\left(v_{2}\right)\right), \mathscr{P}(E)$ as the power set of $E$, and let $C(\cdot)$ satisfy I.P.; then $\langle\cdot, \mathscr{P}(E)\rangle$ forms an abelian semi-group.

Proof: Closure, $v_{1} \cdot v_{2} \in \mathscr{P}(E)$ for all $v_{1}, v_{2} \in \mathscr{P}(E)$, is satisfied since $C(v)$ is defined for all $v$. Commutativity is immediate since $C\left(C\left(v_{1}\right) \cup C\left(v_{2}\right)\right)=C\left(C\left(v_{2}\right) \cup\right.$ $\left.C\left(v_{1}\right)\right)$, i.e., $v_{1} \cdot v_{2}=v_{2} \cdot v_{1}$. We need only establish the remaining propertyassociativity. We seek to show that

$$
v_{i} \cdot\left(v_{j} \cdot v_{k}\right)=\left(v_{i} \cdot v_{j}\right) \cdot v_{k} \quad \text { for all } i, j, k .
$$

First note that application of Theorem 1 and Lemma 1 yields

$$
v \cdot v \equiv C(v) \text {. }
$$

Now for any $v_{i}, v_{j}$, and $v_{k}$, we have

$$
v_{i} \cdot\left(v_{j} \cdot v_{k}\right)=C\left(C\left(v_{i}\right) \cup C\left(C\left(C\left(v_{j}\right) \cup C\left(v_{k}\right)\right)\right)\right) .
$$

Applying I.P., we see $C\left(v_{j} \cup v_{k}\right)=C\left(C\left(v_{j}\right) \cup C\left(v_{k}\right)\right)$ which, when substituted into (8), yields

$$
v_{i} \cdot\left(v_{j} \cdot v_{k}\right)=C\left(C\left(v_{i}\right) \cup C\left(C\left(v_{j} \cup v_{k}\right)\right)\right) .
$$

An application of (7) yields

$$
v_{i} \cdot\left(v_{j} \cdot v_{k}\right)=C\left(C\left(v_{i}\right) \cup C\left(v_{j} \cup v_{k}\right)\right)
$$

and use of I.P. allows us to conclude that

$$
v_{i} \cdot\left(v_{j} \cdot v_{k}\right)=C\left(v_{i} \cup v_{j} \cup v_{k}\right) .
$$

An analogous argument yields

$$
\left(v_{i} \cdot v_{j}\right) \cdot v_{k}=C\left(v_{i} \cup v_{j} \cup v_{k}\right) .
$$

Simply note that (11) and (12) imply (6) and the result is established.

Q.E.D.

We can now move to a consideration of the relationship between path independence and concepts of preference. The following two theorems, Theorems 3 and 4 , use I.P. plus another axiom to characterize a necessary and sufficient condition for a choice function to have a quasi-transitive rationalization. Theorem 5 demonstrates that where the choice is always just one element, path independent choice is equivalent to having a weak order rationalization.

Consider the following axiom:

EXTENSION (E):

$$
(\forall S)_{S \subset E}\left[\left\{x \in S \&(\forall y)_{y \in S} x \in C(\{x, y\})\right\} \Rightarrow x \in C(S)\right] .
$$

The axiom states that if no other element in $S$ beats $x$ in a binary choice, and all such contests are possible, then $x$ is among the chosen elements in $S$. Notice the 
axiom does not imply the existence of a rationalization for the choice, since it does not prohibit elements which $x$ beats in binary contests from being chosen along with $x$. Furthermore the axiom is neither implied by, nor implies I.P. ${ }^{12}$

THEOREM 3: If $C(\cdot)$ satisfies I.P. and E, there exists a unique total, reflexive rationalization $R$ for which $x P y \& y P z \Rightarrow x P z$.

ProOF : Recall, by assumption, that some $V$ contains all subsets of $E$. In particular it contains all two-element subsets. Consequently, the only candidate for a rationalization is the reflexive relation, $R^{*}$, satisfying

$$
\forall x, y_{x, y \in E} x R^{*} y \Leftrightarrow x \in C(\{x, y\}) .
$$

If I.P. and E are satisfied, then $R^{*}$ is a rationalization. To show this we need only establish that

$$
(\forall S)_{S \subset E} C(S)=\left\{x: x \in S \&(\forall y)_{y \in S} x R^{*} y\right\} .
$$

Suppose $x \in C(S)$, and for some $\hat{y}, \hat{y} \in S, \sim x R^{*} \hat{y}$. By definition of $R^{*}$ we have $x \notin C(\{x, \hat{y}\})$. From I.P. we know $C(C([S \backslash\{x, \hat{y}\}]) \cup C(\{x, \hat{y}\}))=C(S)$ but $x \notin$ $C(\{x, \hat{y}\}) \Rightarrow x \notin[C([S \backslash\{x, \hat{y}\}]) \cup C(\{x, \hat{y}\})] \Rightarrow x \notin C(C([S \backslash\{x, \hat{y}\}]) \cup C(\{x, \hat{y}\})) \Rightarrow$ $x \notin C(S)$, contrary to assumption. So $x \in C(S) \Rightarrow x R^{*} y(\forall y)_{y \in S}$. Now if $(\forall y)_{y \in S} x \in$ $C(\{x, y\})$, an application of $E$ yields $x \in C(S)$. So $R^{*}$ is a rationalization.

It remains to be shown that $x P^{*} y \& y P^{*} z \Rightarrow x P^{*} z$. Recalling the definition of $P^{*}$ and $R^{*}$, this property is restated as

$$
y \notin C(\{x, y\}) \& z \notin C(\{y, z\}) \Rightarrow z \notin C\{(x, z\}) .
$$

Assume $y \notin C(\{x, y\}) \& z \notin C(\{y, z\})$. Then

$$
C(\{C(\{y, z\}), x\})=C(\{y, x\})=\{x\}
$$

and

$$
C(\{C(\{x, y\}), z\})=C(\{x, z\}) .
$$

However, from I.P. we know $C(\{C(\{y, z\}), x\})=C(\{C(\{x, y\}), z\})$ which implies $C(\{x, z\})=x$. We conclude that $z \notin C(\{x, z\})$ which completes the proof. Q.E.D.

THEOREM 4: If $R$ is any total, reflexive, binary relation over $E$ for which $x P y \&$ $y P z \Rightarrow x P z$, then $R$ induces a unique choice function and this function satisfies I.P. and E.

Proof : That an $R$ with the above properties induces a choice function is shown in $[14$, Theorem II]. Since $R$ is total and reflexive it is consistent with one and only one choice function-call this function $C(\cdot)$. We need only show that $C(\cdot)$ satisfies I.P.

${ }^{12}$ Both Fishburn [7] and Sen [16] have introduced axioms similar to this one. Both, however, differ from each other and differ from this axiom. Sen's is introduced below as his $\gamma$. 
and E. By the definition of "induced,"

$$
C(S)=\left\{x: x \in S \& \forall y_{y \in S} x R y\right\},
$$

we see $E$ is satisfied.

Now we need to show only that

$$
(\forall S)_{S \subset E}(\forall V)_{V \in V S} C(S)=C\left(\bigcup_{v \in V} C(v)\right) .
$$

We show first that $C(S) \subset C\left(\bigcup_{v \in V} C(v)\right)$ for any $S \subset E$ and $V \in V_{S}$. If $\hat{x} \in C(S)$, we know, by definition of $C(\cdot)$, that $(\forall y)_{y \in S} \hat{x} R y$. It follows directly that $(\forall v)_{v} \hat{x} \in \hat{x} \in v \Rightarrow$ $\hat{x} \in C(v)$. We know now that, for any $\left(v_{1}, \ldots, v_{m}\right)$ with $v_{i} \subset S$, if $\hat{x} \in \cup_{i} v_{i}$, then $\hat{x} \in \cup_{i} C\left(v_{i}\right)$. But since $\cup_{i} C\left(v_{i}\right) \subset S$ and $\hat{x} \in \cup_{i} C\left(v_{i}\right)$, we have $\hat{x} \in C\left(\cup_{i} C\left(v_{i}\right)\right)$ as asserted.

We now show

$$
(\forall V)_{V \in V S} C\left(\bigcup_{v \in V} C(v)\right) \subset C(S) .
$$

Suppose $\hat{x} \notin C(S)$. Then $(\exists \hat{y})_{\hat{y} \in S}$ such that $\sim \hat{x} R \hat{y}$ since by construction $C(S)$ is the set of $R$-maximal elements of $S$. Assume further $\hat{x} \in C\left(\bigcup_{v \in V^{*}} C(v)\right)$ for some $V^{*} \in$ $V_{S}$. From this we get $\hat{x} \in \bigcup_{v \in V^{*}} C(v) \Rightarrow \hat{y} \notin \bigcup_{v \in V^{*}} C(v) \Rightarrow\left[\hat{y} \in v \Rightarrow(\exists z)_{z \in V^{*}} z \hat{y}\right]$. Since $\cup v=S$ and $\hat{y} \in S$ we know $\hat{y} \in v$ for some $v_{0}$, and by the previous sentence there is a $z_{1} \in v_{0}$ such that $z_{1} P \hat{y}$. But $z_{1} \notin C\left(v_{0}\right)$, for if it were, then $z_{1} \in \bigcup_{v \in V^{*}} C(v)$, but since $z_{1} P \hat{y} \& \hat{y} P \hat{x}$ by transitivity of $P$, we conclude $z_{1} P \hat{x}$ which implies $\hat{x} \notin$ $C\left(\bigcup_{v \in V^{*}} C(v)\right)$, as assumed. Therefore, $z_{1} \notin C\left(v_{0}\right)$, so there exists a $z_{2} \in v_{0}$ such that $z_{2} P z_{1}$. The same argument yields $z_{2} \notin C\left(v_{0}\right)$ and the existence of a $z_{3}$ in $v_{0}$ such that $z_{3} P z_{2}$. By the finiteness of $E$ the argument can be repeated until the elements in $v_{0}$ are exhausted, implying $C\left(v_{0}\right)=\varnothing$, contrary to assumption. We reject the assumption $\hat{x} \in C\left(\bigcup_{v \in V^{*}} C(v)\right)$ and conclude

$$
\hat{x} \notin C(S) \Rightarrow \hat{x} \notin C\left(\bigcup_{v \in V} C(v)\right) \quad \text { for any } V \in V_{S},
$$

or,

$$
C(S) \supset C\left(\bigcup_{v \in V} C(v)\right)
$$

THEOREM 5: If choice over any set is always a single element, and $C(\cdot)$ satisfies I.P., then there exists a unique, total, reflexive, transitive rationalization.

Proof: Suppose $\{x\}=C(\{x, y\}) \& y=C(\{z, y\})$. If I.P. is satisfied, we note $x=C(\{x, C(\{y, z\})\})=C(\{C(\{x, y\}), z\})=C(\{x, z\})$. So binary choice is transitive. Define $x P y$ in case $x=C(\{x, y\})$ and note $P$ is a transitive relation. Define now $x R y \Leftrightarrow[x P y$ or $x=y]$ and note $R$ is a total, reflexive, transitive relation. Q.E.D. 


\section{5. "HISTORY-FREE" CHOICE}

The second class of motivations for path independence stems from a different but less precise demand when compared to the discussion above. Arrow's original mention of path independence occurs when he is discussing biases in favor of the status quo which, itself, could be the result of the accidental forces of history. He sees "social deadlock" or "paralysis" [6] as something to be avoided in favor of "full adaptability to varying environments" [2, p. 120]. The status quo, or history, should play no dominant role in the determination of choice.

This is a most perplexing argument since the simplest way to assure that the status quo is not favored is to make sure that the choicefunction is not parameterized by such a variable. Such parameters can be introduced $[\mathbf{1 0}]$, but they have absolutely nothing to do with rational choice or path independence. The perplexing thing is that no such parameters ever appear in Arrow. The choice from a set is always independent of the status quo in his formulation. That is, in the Arrow formulation the choice from a given set can be the same regardless of the alternative indexed as the status quo and regardless of whether or not the process satisfies some type of rationality condition.

That said, however, the matter can still be pursued. Suppose a social choice process is viewed as "working its way" through the elements of $v$ and then "choosing." Suppose the agenda is changed from $v_{1}$ to $v_{2}$. Since all of the elements of $v_{1}$ have been "examined," it would seem "rational" to eliminate the problem of "examining" and "considering" those elements of $v_{1}$ still available, as well as the new ones, in order to determine $C\left(v_{2}\right)$. We could view the reason for this requirement as being some type of "computational efficiency" argument.

Two separate axioms are offered as formalizations of this rather loose idea. The reader should also consider I.P." with these interpretations in mind.

Axiom 1: $\left(\forall v_{1}\right)_{v_{1} \subset E}\left(\forall v_{2}\right)_{v_{2} \subset E} C\left(C\left(v_{2} \backslash v_{1}\right) \cup C\left(v_{2} \cap v_{1}\right)\right)=C\left(v_{2}\right)$.

This axiom says that if the agenda changes from $v_{1}$ to $v_{2}$, the choice over $v_{2}$ can be computed by first considering the set remaining of what was previously available, and choosing. Then one takes the chosen elements $C\left(v_{1} \cap v_{2}\right)$, together with choice over the new elements, $C\left(v_{2} \backslash v_{1}\right)$, and chooses over the union of the chosen elements. The result $C\left(C\left(v_{2} \backslash v_{1}\right) \cup C\left(v_{2} \cap v_{1}\right)\right)$ should be the same as $C\left(v_{2}\right)$.

Axiom 1 is very "close" to I.P.', and thus it is "close" to I.P. However, it still differs in an important way. Axiom 1 says that if you take a set and partition it into two parts, the outcome of the resulting sequence of choices is independent of the particular partition; whereas I.P.' is exactly the same except that the two initial subsets need not necessarily form a partition - the sets might "overlap."

Remark 3 : (i) I.P. implies Axiom 1; (ii) Axiom 1 does not imply I.P.

Proof: The truth of (i) is obvious since Axiom 1 is simply the restriction of I.P. to disjoint paths. In order to establish (ii) we need only consider an example. ${ }^{13}$

${ }^{13} \mathrm{~A}$ more interesting example can be found in $[11]$. 
Let $C(\alpha)=\alpha$ for $\alpha \in[\{x, y\},\{x, z\},\{y, z\}]$ and let $C(\{x, y, z\})=\{x\}$. I.P. is not satisfied since for $V=(\{x, y, z\},\{y\})$ we have $C(C(\{x, y, z\}) \cup C(\{y\}))=\{x, y\} \neq$ $C(\{x, y, z\})$. The reader can easily establish that the example satisfies Axiom 1 .

Q.E.D.

We can, with the aid of the following remark and with the aid of results provided by others, indicate how Axiom 1 is connected to concepts of preference.

REMARK 4: Axiom 1 implies but is not equivalent to the following axiom:

$$
(\forall S)_{S \subset E}(\forall V)_{V \in V_{S}}\left[C(S) \subset C\left(\bigcup_{v \in V} C(v)\right)\right] .
$$

Proof: Suppose, for some $V \in V_{S}$ we have $x \notin C\left(\bigcup_{v \in V} C(v)\right)$ but $x \in C(S)$. If $x \in \bigcup_{v \in V} C(v)$, we let $S_{1}=\bigcup_{v \in V} C(v)$ and $S_{2}=S$. Since $x \notin S_{2} \backslash S_{1}$ and $x \notin C\left(S_{1}\right)$, $x \notin C\left(C\left(S_{2} \backslash S_{1}\right) \cup C\left(S_{1} \cap S_{2}=S_{1}\right)\right)$ so Axiom 1 is violated. If $x \notin \bigcup_{v \in V} C(v)$, consider $v_{0}$ with $v_{0} \in V$ and $x \in v_{0}$. Such a $v_{0}$ exists by construction. Now $x \notin C\left(v_{0}\right)$ by assumption. Letting $S_{1}=v_{0}$ and $S_{2}=S$, we have $C\left(C\left(S_{2} \backslash S_{1}\right) \cup C\left(S_{2} \cap S_{1}=\right.\right.$ $\left.\left.S_{1}\right)\right) \neq C(S)$, in violation of Axiom 1. The following example satisfies the statement contained in the Remark but does not satisfy Axiom 1. Hence Axiom 1 implies the statement but is not equivalent to it.

Consider $C(\{x, y\})=\{x, y\}, C(\{y, z\})=\{y\}, C(\{x, z\})=\{x\}, C(\{x, y, z\})=\{x\}$. Note $C(\{C(\{x, y\}), z\})=\{x\}, C(\{C(\{y, z\}), x\})=\{x, y\}, C(\{C(\{x, z\}), y\})=\{x, y\}$ suggests that $C(\{x, y, z\})$ is contained in the choice over every path. It is easy to prove that it is so, as follows. Note that for the example, (i) $[x \in v \& v \subset\{x, y, z\}] \Rightarrow$ $x \in C(v)$, and (ii) $\left[\bigcup_{v \in V} v=\{x, y, z\}\right] \Rightarrow\left(\exists v_{0}\right)_{v_{0} \in V}$ such that $x \in v_{0}$. These two statements imply that $x \in \bigcup_{v \in V} C(v)$. But then by (i) and the construction of $C(\{x, y, z\})$ we have $C(\{x, y, z\})=x \in C\left(\bigcup_{v \in V} C(v)\right)$. The example thus satisfies the conditions of axiom (*). Axiom 1 is not satisfied, however, since $C(\{x, \dot{y}, z\}) \neq$ $C(C(\{x, z\}) \cup C(\{y\}))$.

Q.E.D.

In order to connect Axiom 1 to concepts of preference, the reader should consult Parks [8] and Sen [16]. Parks establishes that axiom (*) stated in Remark 4 is equivalent to an axiom Sen calls $\alpha$. Sen, in turn, shows that $\alpha$, plus an axiom he terms $\gamma,{ }^{14}$ together are necessary and sufficient for a choice function to have a rationalization; that is, these two axioms, together, are equivalent to Richter's $V$ axiom [13], when the latter is restricted to all finite sets. So, if axiom $(*)$ in Remark 4 is appended to $\gamma$, a characterization of rational choice is obtained. If, however, Axiom 1 is appended to $\gamma$, we obtain, in addition to those of the previous section, another characterization of quasi-transitive rational choice. This second result is presented as the following theorem.

THEOREM 6: A choice function satisfies Axiom 1 and $\gamma$ if and only if it satisfies I.P. and $E$.

${ }^{14}$ The axioms are as follows, where the sets $v_{1}$ and $v_{2}$ are arbitrary.

Ахіом $\alpha: v_{1} \subset v_{2} \Rightarrow\left[x \in C\left(v_{2}\right) \cap v_{1} \Rightarrow x \in C\left(v_{1}\right)\right]$.

Ахіом $\gamma: V \in V_{S} \Rightarrow\left[\bigcap_{v \in V} C(v) \subset C(S)\right]$. 
Proof: We show first that [Axiom $1 \& \gamma] \Rightarrow$ [I.P. \& E]. Notice that if Axiom 1 and $\gamma$ are satisfied then $C(\cdot)$ is rational. This follows directly from Remark 4 together with the Parks-Richter-Sen results. Furthermore, the $P$ relation induced by the rationalization must be transitive. If it is not transitive, then for some $x, y$, and $z$ we have either $x P y \& y P z \& z P x$ which is impossible since it would imply, contrary to assumption, that $C(\{x, y, z\})=\varnothing$, or $x P y \& y P z \& z R x$. Suppose the latter were true. Then let $v_{2}=\{x, y, z\}$ and $v_{1}=\{z\}$ and note $C(C(\{x, y\}) \cup C(\{z\}))=$ $\{x, z\} \neq\{x\}=C(\{x, y, z\})=C\left(v_{2}\right)$ which contradicts Axiom 1. So the induced $P$ relation must be transitive. Now, since we know $C(\cdot)$ must be rational with a transitive $P$, we apply Theorem 4 and conclude directly that I.P. and $\mathrm{E}$ are both satisfied.

Now suppose I.P. and E are satisfied. Clearly Axiom 1 is satisfied. Application of Theorem 3 indicates that $C(\cdot)$ is rational and application of Sen $[\mathbf{1 6}$, Theorem T.9] indicates that $\gamma$ is satisfied.

Q.E.D.

We return now to the ideas which motivated this section and present one final axiom. Suppose we demand that the only information one retains about the current agenda is the choice. If the agenda changes, we might demand the new choice be determined in a contest between previous choices still available and the new opportunities.

Formally, the idea is stated as follows:

Aхіом 2:

$$
\left(\forall v_{1}\right)_{v_{1} \subset E}\left(\forall v_{2}\right)_{v_{2} \subset E}\left[\left\{\left[C\left(v_{1}\right) \cap v_{2}\right] \neq \varnothing\right\} \Rightarrow\left\{C\left(\left[v_{2} \backslash v_{1}\right] \cup\left[C\left(v_{1}\right) \cap v_{2}\right]\right)=C\left(v_{2}\right)\right\}\right] .
$$

The following results :

THEOREM 7: If $C(\cdot)$ satisfies Axiom 2, there exists a unique, total, reflexive, transitive binary relation which rationalizes the choice. Furthermore, every total, reflexive, transitive binary relation induces a choice function which satisfies Axiom 2.

Proof: The result could be established by showing the axiom is equivalent to the weak axiom of revealed preference which in turn implies, under the hypotheses employed here, the existence of a unique weak order rationalization $[\mathbf{1}$, Theorem 3]. Instead, we will establish the first result independently.

Assume first $x \in C(\{x, y\}) \& y \in C(\{y, z\})$. If $x \notin C(\{x, y, z\})$, then $y \notin C(\{x, y, z\})$, since if $y \in C(\{x, y, z\})$ we use the axiom and

$$
C(\{x, y\})=C([\{x, y\} \backslash\{x, y, z\}] \cup[C(\{x, y, z\}) \cap\{x, y\}])=C(y)=y,
$$

contrary to assumption. Furthermore, if $x \notin C(\{x, y, z\})$, then $z \notin C(\{x, y, z\})$, since if it were the axiom implies, contrary to assumption,

$$
C(\{y, z\})=C([\{y, z\} \backslash\{x, y, z\}] \cup[\{z\} \cap\{y, z\}])=\{z\} .
$$

Since $C(\{x, y, z\}) \neq \varnothing$, we conclude $x \in C(\{x, y, z\})$. Now if $x \notin C(\{x, z\})$, from the axiom we obtain

$$
C(\{x, y, z\})=C([\{x, y, z\} \backslash\{x, z\}] \cup[\{x, y, z\} \cap\{z\}])=C(\{y, z\}),
$$

contrary to the conclusion that $x \in C(\{x, y, z\})$. So, $x \in C(\{x, z\})$. 
Define $\gamma R \delta$ if and only if $\gamma \in C(\{\gamma, \delta\})$. Since $C(\cdot)$ is defined for all two-element sets, $R$ is total and reflexive (by convention). From the above result $R$ is transitive. We show $R$ "rationalizes" $C(\cdot)$.

Suppose $x \in C(S)$ but for some $y \in S, x \notin C(\{x, y\})$. We then get, contrary to the axiom, $x \notin C(C(S \backslash\{x, y\}) \cup[S \cap C(\{x, y\})])$. Suppose now $x \in C(\{x, y\})$ for all $y$ in $S$ but $x \notin C(S)$. Now, $\{x, \hat{y}\} \cap C(S) \neq \varnothing$ for some $\hat{y}$ since $C(S) \neq \varnothing$, and from the axiom we get $C(\{x, \hat{y}\})=C([\{x, \hat{y}\} \backslash S] \cup[\{x, \hat{y}\} \cap C(S)])$. Now $(\{x, \hat{y}\} \backslash S)=\varnothing$. Furthermore, $x \notin[\{x, \hat{y}\} \cap C(S)]$ by assumption, so $x \notin[C(\{x, \hat{y}\} \backslash S) \cup[\{x, \hat{y}\} \cap$ $C(S)]]$; thus we get

$$
x \notin C([\{x, \hat{y}\} \backslash S] \cup[\{x, \hat{y}\} \cap C(S)])=C(\{x, \hat{y}\})
$$

in violation of our assumption. We conclude $x \in C(S)$ if $x \in C(\{x, y\})$ for all $y \in S$ but since $x \in C(\{x, y\})$ if $x R y$, we conclude $R$ is a rationalization. Proof of the second half is left to the reader.

Q.E.D.

\section{IMPOSSIBILITY RESULTS}

We can now turn to a consideration of Arrow's major theorem. One of the conditions he requires in his structure is that the social choice function must be total, reflexive, transitive, and rational. The various motivations for this restriction have been reviewed above.

If we replace his consistency conditions with I.P., his impossibility results do not follow. In fact, we can, in addition to I.P., require $\mathrm{E}$ without an impossibility result. An example is provided by Sen [14, Theorem V] which, in view of Theorem 4 here, has the requisite properties.

The example given by Sen is the process that always chooses all Pareto optimal alternatives. Such a process has a quasi-transitive rationalization and thus satisfies I.P. and E. Of course, to some this might not be a very exciting example. Notice, however, that there is no need of requiring the property $\mathrm{E}$, and the removal of this admits additional examples. In fact, there appears to be no overriding reason to impose even I.P. at the very outset of the analysis, even though special considerations make I.P. appear to be a potentially useful tool.

The point is, then, that the path independent line of argument yields no general support for the imposition of rationality conditions on social choice. Support for the rationality condition must be based on some other line of argument, such as one of those outlined in the first section. If no real justification resides there either, then the obvious thing to do is drop the concept of social rationality and apply the tools that remain toward some positive end.

\section{California Institute of Technology}




\section{GLOSSARY}

$(\forall x)_{x \in y}$ means: For all $x$, if $x \in Y$, then ...

$(\exists x)_{x \in y}$ means: There exists an $x$ such that $x \in Y$ and $\ldots$

$\sim$ means: It is not true that....

$\{x: \ldots\}$ means: The set of $x$ such that ....

$A \cap B=\{x: x \in A \& x \in B\}$.

$A \cup B=\{x: x \in A$, or $x \in B$, or both $\}$.

A binary relation $R$ on a set $A$ is :

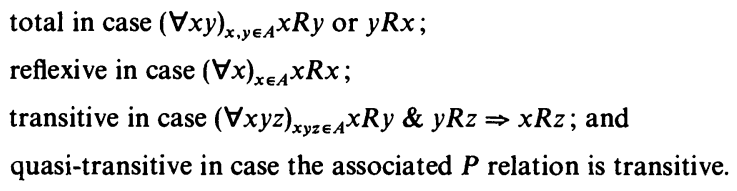

A total, reflexive, transitive binary relation is called a weak order.

A binary relation $R$ induces the choice function $C(S)$ defined by

$$
C(S)=\left\{x: x \in S \&(\forall y)_{y \in S} x R y\right\} .
$$

A choice structure $\langle V, C(S)\rangle$ is rationalized by a binary relation $R$ in case

$$
(\forall S)_{S \in V} C(S)=\left\{x: x \in S \&(\forall y)_{y \in S} x R y\right\} .
$$

\section{REFERENCES}

[1] Arrow, K. J.: "Rational Choice Functions and Orderings," Economica, 26 (1959), 121-127.

[2] —-: Social Choice and Individual Values. 2nd ed. New York: Wiley, 1963.

[3] BLAU, J.: "The Existence of Social Welfare Functions," Econometrica, 25 (1957), 302-313.

[4] Buchanan, J. M.: "Individual Choice in Voting and the Market," Journal of Political Economy, 62 (1954), 334-343.

[5] Buchanan, J. M., AND G. Tullock: The Calculus of Consent. Ann Arbor: University of Michigan Press, 1962.

[6] DAHL, R.: A Preface to Democratic Theory. Chicago: University of Chicago Press, 1956.

[7] FISHBURN, P. C.: "Irrationality of Transitivity in Social Choice," Behavioral Science, 15 (1970), 119-123.

[8] PARKs, R. P.: "Rationalizations, Extensions, and Social Choice Paths," mimeographed, Washington University, St. Louis, 1971.

[9] PLotT, C. R.: "Recent Results in the Theory of Voting," in Frontiers of Quantitative Economics, Michael D. Intriligator, ed. Amsterdam: North-Holland Publishing Co., 1971.

[10] - "Individual Choice of a Decision Process," in Probability Models of Collective Decision Making, R. Niemi \& H. Weisberg, eds. Columbus, Ohio: Charles E. Merill Publishing Co., 1972.

[11] _ - "Social Choice and Social Rationality," Social Science Working Paper No. 2, California Institute of Technology, Pasadena, Calif., 1971.

[12] Richter, M.: "Revealed Preference Theory," Econometrica, 34 (1966), 635-645.

[13] — : "Rational Choice," in Preferences, Utility and Demand, John Chipman et al., eds. New York: Harcourt, Brace, Jovanovich, 1971.

[14] SeN, A. K.: "Quasi-transitivity, Rational Choice and Collective Decisions," Review of Economic Studies, 36 (1969), 381-393.

[15] - Collective Choice and Social Welfare. San Francisco: Holden-Day. 1970.

[16] - "Choice Functions and Revealed Preference," Review of Economic Studies, 38 (1971), 307-317.

[17] WiLson, R.: "Stable Coalition Proposals in Majority-Rule Voting," Journal of Economic Theory, 3 (1971), 254-271.

[18] — : "A Game-Theoretic Analysis of Social Choice," in Social Choice, B. Lieberman, ed. New York: Gordon and Breach, 1971. 